\title{
Forward modeling for marine sediment characterization using chirp sonars
}

\author{
Sandrine Rakotonarivo ${ }^{1}$, Michel Legris ${ }^{2}$, Rozenn Desmare ${ }^{3}$, Jean-Pierre Sessarégo ${ }^{4}$ \\ and Jean-François Bourillet ${ }^{5}$
}

\begin{abstract}
This paper investigates the forward modeling of chirpsonar data for the quantitative characterization of marine subbottom sediment between 1 and $10 \mathrm{kHz}$. The forward modeling, based on a transfer function approach, included impacts of layering or impedance mismatch, attenuation, roughness, and transitional layers, i.e., continuous impedance variations. The presented approach provided the best compromise between the number of available geoacoustic parameters from chirp-sonar data and the subbottom modeling accuracy. The forward model was tested on deep-sea chirp-sonar data acquired at a central frequency of $3.5 \mathrm{kHz}$. Comparisons between synthetic and experimental seismograms showed good agreement for the first $15 \mathrm{~m}$ of buried layers. Performance of the inversion using this forward model was also examined through sensitivity analysis. The results suggested that estimations of layer thickness, impedance, and transitional layer thickness were robust, whereas roughness and attenuation estimations were subject to wavelength and layer thickness conditions
\end{abstract}

\section{INTRODUCTION}

Quantitative marine-sediment characterization using acoustic waves has emerged as a major area of research for the petroleum industry (Stoll and Bryan, 1979; Hardage et al., 2006). The technique is also increasingly applied to technical opera- tions such as harbor dredging, sand and gravel extraction, and site surveys for telecommunication or electrical power cables.

The remote sensing of subbottom sediment requires two fundamental conditions: (1) access to broadband, calibrated, energetic signals for quantitative signal processing and (2) a theoretical model for subbottom parameter estimation and sediment classification. Present-day chirp sonars, typically working at frequencies between 1 and $10 \mathrm{kHz}$, can image as much as the first hundred meters of soft marine sediment. Such devices provide repeatable, calibrated, broadband chirp signals with a wide range of design possibilities (Schock et al., 1989; Gutowski et al., 2002; Langli and LeGac, 2004) and enable quantitative estimations of acoustic sediment parameters. These parameters, known as geoacoustic parameters, can be further analyzed to derive other sediment parameters (e.g., porosity, mean grain size) (Hamilton, 1972; Hamilton and Bachman, 1982) and to classify the subbottom sediment.

However, the single sensor pair and the single incidence angle configuration of chirp sonars limit the number of accessible sediment parameters from acoustic data. Therefore, remote sediment sensing using chirp sonars requires the use of relatively simple forward modeling with a small number of parameters as opposed to the large number of parameters needed to describe the complexity of subbottom sediments. Indeed, the modeling of acoustic wave propagation in marine sediment (Chotiros and Isakson, 2004; Buckingham, 2007) remains a challenging problem. Subbottom sediments are absorbing, layered, porous, and scattering media. They may contain rough interfaces, discrete scatterers (e.g., shells, rocks), and continuous impedance variations (e.g., effect of consolidation on sandy/muddy sediments, transition layers between water and sediment, turbidites). We refer to layers

\footnotetext{
Manuscript received by the Editor 2 June 2010; revised manuscript received 20 January 2011; published online 13 June 2011.

${ }^{1}$ Formerly Université Européenne de Bretagne, École Nationale Supérieure de Techniques Avancées (ENSTA) Bretagne, Laboratoire Extraction et Exploitation de l'Information en Environnements Incertains, Brest, France; presently University of California San Diego, Scripps Institution of Oceanography, Marine Physical Laboratory, La Jolla, California, U.S.A. E-mail: srakotonarivo@ucsd.edu.

'Université Européenne de Bretagne, École Nationale Supérieure de Techniques Avancées (ENSTA) Bretagne, Laboratoire Extraction et Exploitation

de 1'Information en Environnements Incertains, Brest, France. E-mail: michel.legris@ensieta.fr.

${ }^{3}$ Institut de Recherche de l'École Navale (IRENav), Brest, France. E-mail: rozenn.desmare@ecole-navale.fr.

${ }_{5}^{4}$ Centre National de la Recherche Scientifique, Laboratoire de Mécanique et d'Acoustique (LMA), Marseille, France. E-mail: sessarego@lma.cnrs-mrs.fr.

${ }^{5}$ Institut Français de Recherche pour l'Exploitation de la Mer (IFREMER), Unité de recherche Géosciences Marines, Plouzane, France. E-mail: Jean.Francois.Bourillet@ifremer.fr.

(C) 2011 Society of Exploration Geophysicists. All rights reserved.
} 
with continuous impedance variation as transitional layers in this paper.

In that context, the first motivation of this study is to maximize the accuracy of the forward modeling with respect to the chirp-sonar configuration and to the subbottom sediment complexity. Three sediment parameters might be determined directly from the coherent component of chirp-sonar data (Panda et al., 1994; Schock, 2004a, 2004b): marine-sediment attenuation (LeBlanc et al., 1992a; Stevenson et al., 2002; Pinson et al., 2008; Theuillon et al., 2008), reflection loss or impedance (LeBlanc et al., 1992b; DeBruin, 1995; Bull et al., 1998), and layer thickness (in terms of two-way traveltime). Those approaches do not estimate or include impacts of roughness and transitional layers in the forward modeling, whereas they may disturb the wave propagation and impact the quality of subbottom parameter estimations. Also, chirp signals often penetrate more than one layer into the sea bottom. However, most previous work has estimated sediment parameters of the first subbottom sediment layer. Consequently, this paper addresses the forward modeling of chirp-sonar data to improve the performance of the inversion for characterizing deeper subbottom sediment layers. The approach consists of modeling the coherent wave forward propagation using the transfer function formalism (Brekhovskikh and Lysanov, 1991; Panda et al., 1994) and of incorporating the effects of roughness and transitional layers.

A second motivation of this work is to analyze the extent to which sediment parameters can be extracted from chirp-sonar data through forward modeling. The inversion will consist of minimizing a cost function that quantifies the bias between the model and the data. Therefore, we examine the effect of each sediment parameter on the output of the cost function. Through sensitivity analysis, robustness of sediment parameter estimation

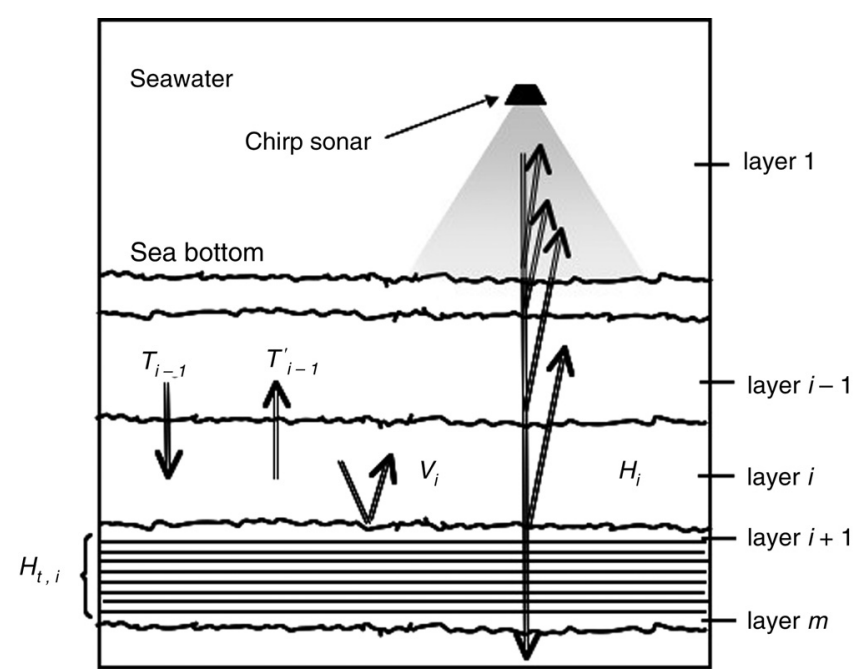

Figure 1. Synoptic diagram of the chirp-sonar setup. The chirp sonar transmits a wave into the subbottom sediment and measures the wave reflected back by the subbottom. The subbottom sediment is described as a layered medium. Each layer is characterized by its transfer function $H_{i}$, and interfaces are identified by the forward and backward transmission coefficients, $T_{i-1}$ and $T_{i-1}^{\prime}$, and the reflection coefficient $V_{i}$. Transitional layers or continuous impedance variations are described by their transfer function $H_{t, i}$. They are discretized into $M$ sublayers with constant values of sediment parameters inside each sublayer. is tested versus frequency range, subbottom configuration, and depth below seafloor. The inverse problem is not treated here.

The structure of the paper is as follows. The first section presents the theoretical basis of the forward modeling based on a transfer function approach and justifies the mathematical description of attenuation, transitional layer, and roughness. The forward modeling is tested on chirp-sonar data and a core sample from deep-sea measurements in situ. The following section conducts a sensitivity analysis of the forward modeling for two subbottom sediment scenarios. Finally, the inversion performances are discussed and conclusions are presented.

\section{FORWARD MODELING}

Chirp sonars work at normal incidence in a monostatic configuration. The typical operating frequencies range from 1 to 10 $\mathrm{kHz}$. Sonar antennas transmit a broadband chirp that propagates vertically toward the sea bottom (Figure 1) and receive the wave reflected back by the subbottom sediment. Acoustic data measured by chirp sonars can be expressed as

$$
y_{j}(t)=e(t) * i(t) * p_{j}(t),
$$

where $y_{j}(t)$ describes the acoustic signal measured by the chirp sonar, $e(t)$ is the transmitted chirp, $i(t)$ represents the instrument response of the measurement chain (amplifiers, transducers sensitivity, and impedance matching), $t$ is time, and $j$ denotes the observation index. Also, the pressure response $p_{j}(t)$ includes the effects of seawater and of the subbottom sediment. The asterisk (*) stands for the convolution product.

The acoustic signal is crosscorrelated to the transmitted chirp to increase the gain (Schock et al., 1989): $\gamma_{j}(\tau)=y_{j}(t) * e(t-\tau)$. This matched-filtering operation enables sending long-duration signals, i.e., more energy, to the subbottom sediment while keeping a resolution $\delta \tau=1 / B_{w}$, which only depends on the chirp bandwidth $B_{w}$ and not on the chirp duration. We refer to the crosscorrelated signal as the compressed seismogram in this paper.

Assuming low statistic spatial variability of the sea bottom over $N$ acquisitions, remote sensing of the subbottom sediment is performed on the coherent average of the compressed seismogram over spatial locations, $\gamma=1 / N \sum_{j=1}^{N} \gamma_{j}$. We also assume that acoustic data are calibrated, i.e., the instrument response is known, so that only the coherent response including the effects of seawater and of the subbottom sediment has to be modeled:

$$
p(t)=\frac{1}{N} \sum_{j=1}^{N} p_{j}(t) .
$$

In the following, the subbottom sediment is described (Figure 1) as a fluid-attenuated layered medium with rough interfaces and transitional layers. Due to the low shear-wave velocity (Ivakin and Jackson, 1998) and the low conversion rate of compressional waves into shear waves for wave propagation close to the normal incidence (Brekhovskikh and Lysanov, 1991), the seabottom sediment is suitably represented as a fluid medium. We only consider layers that are parallel to each other because the beam pattern of chirp sonars is more sensitive to perpendicular layers (Langli and LeGac, 2004). Multiple reflections inside the subbottom sediment are neglected due to the presence of attenuation in marine sediment (Hamilton, 1972; Bowles, 1997) and to low-impedance contrasts. Indeed, typical values of sound 
speed and density in soft marine sediment (Hamilton, 1970; Hamilton and Bachman, 1982) result in impedance values between $150 \times 10^{4}$ and $378 \times 10^{4} \mathrm{~Pa}-\mathrm{s} / \mathrm{m}$.

Because of the wide aperture of chirp-sonar antennas, we assume spherical spreading. For example, Echoes 3500 profilers manufactured by IXSEA have a half directivity (defined at $-3 \mathrm{~dB}$ ) of $30^{\circ}$ at $3.5 \mathrm{kHz}$. Assuming that the chirp sonar is located at many wavelengths from the sea bottom and following the transfer function method (Brekhovskikh and Lysanov, 1991; LeBlanc et al., 1992b; Panda et al., 1994), the coherent wave propagation into seawater and the subbottom sediment is modeled as

$\tilde{p}(\omega)=p_{0} D_{\theta}^{2}\left[\frac{e^{-j \omega \tau_{1}}}{c_{1} \tau_{1}} V_{1}+\sum_{i=2}^{n-1}\left(\frac{e^{-j \omega \tau_{i}}}{c_{1} \tau_{i}} V_{i} \prod_{m=2}^{i} H_{m}^{2} T_{m-1} T_{m-1}^{\prime}\right)\right]$,

where $\tilde{p}(\omega)$ is the acoustic transfer function at frequency $\omega, p_{0}$ is the initial incident pressure amplitude, $D_{\theta}$ represents the directivity function (defined in amplitude), $\tau_{i}$ is the two-way traveltime from the sonar position to the layer $i$, and $n$ is the number of layers. The term $V_{i}$ is the coefficient of reflection, and $T_{i}$ and $T_{i}^{\prime}$ describe the coefficients of transmission in the forward and backward directions, respectively. Those coefficients comprise the effects of layering and of roughness that are discussed below. The transfer function of the layer $i, H_{i}$, contains the effect of attenuation that is presented in the next section. The denominator $c_{1} \tau_{i}$ quantifies geometrical spreading, where $c_{1}$ is the sound speed in seawater. Because of the operational configuration of chirp sonars, the sound speed in the sediment cannot be retrieved. Therefore, the sound speed in the sediment is approximated by the seawater sound speed. This approximation produces less than 0.2-dB error per layer, as discussed by Rakotonarivo (2009). The impulse response in equation 2 is related to the acoustic transfer function in equation 3 through the inverse Fourier transform:

$$
p(t)=\frac{1}{2 \pi} \int_{-\infty}^{+\infty} \tilde{p}(\omega) e^{j \omega t} d \omega
$$

The forward model presented in this section incorporates five sediment parameters: the two-way traveltime from the sonar to the sediment layers, the impedance and the roughness through transmission and reflection coefficients, the attenuation through the layer transfer function, and the transitional layer. The following sections describe how the attenuation, the transitional layer, and the roughness are included in the forward model.

\section{Attenuation}

The transfer function given in equation 3 comprises effective sediment attenuation caused by intrinsic absorption and volumetric scattering losses (Jacobson, 1987):

$$
\begin{gathered}
H_{i}(\omega)=e^{-\alpha_{i}(\omega) c_{1} \frac{\tau_{i}-\tau_{i-1}}{2}}, \\
\alpha_{i}(\omega)=\frac{\ln (10)}{20}(\omega / 2 \pi)^{n_{i}} k_{p, i},
\end{gathered}
$$

where $\alpha_{i}$ is the attenuation factor (in $\mathrm{Np} / \mathrm{m}$ ) in layer $i$. The attenuation coefficient $k_{p, i}$ and the exponent $n_{i}$ depend on the sediment description and the frequency. At the present time, neither mechanisms nor mathematical descriptions of attenuation are precisely known to determine the attenuation coefficient and the exponent. Viscoelastic models assume that anelastic frame losses are the dominant dissipative process in marine sediment and state that the attenuation of compressional waves varies with the first power of frequency (Hamilton, 1972; Buckingham, 2007). Alternatively, the Biot-Stoll theory (Stoll, 1977; Chotiros and Isakson, 2004) associates attenuation with the movement of pore fluids. It predicts a nonlinear attenuation law with frequency that is compatible with attenuation data discussed by Kibblewhite (1989). To provide a forward modeling with the best match with ground-truth data, we adopt an empirical approach to justify the attenuation model used in this study. Consequently, attenuation mechanisms are not discussed in the paper. There is a wide range of literature on this topic, and the reader can refer to the work of previous studies (Hamilton, 1972; Kibblewhite, 1989; Stoll, 1977) for further discussions of attenuation in marine sediment.

Table 1 summarizes attenuation measured in situ for many sediment types at frequencies overlapping the band $1-10 \mathrm{kHz}$. Most attenuation coefficients $k_{p}$ and exponents $n$ given in Table 1 converge toward a linear frequency-dependent attenuation. Given this observation, we have applied a regression analysis to each of the values of exponent and attenuation coefficients given in Table 1. For all sediment types in Table 1, the regression provides a coefficient $k_{p}^{\prime} \in[0.076 ; 0.79]$ and an offset $b^{\prime} \in$ $[-0.24 ; 2.52]$ with a linear correlation coefficient $R^{2}$ close to unity, $R^{2}>0.98$. This justifies the use of a model supporting a linear frequency-dependent attenuation at frequencies ranging from 1 to $10 \mathrm{kHz}$. As the offset $b^{\prime}$ is close to zero for most sediment attenuations in Table 1, the latter is neglected. Thus, marine-sediment attenuation is characterized by one parameter in this study: the attenuation coefficient.

\section{Transitional layer}

To model the impact of continuous variation of impedance, the transitional layer is discretized into $M$ layers. Following the transfer function method of equation 3 , the transfer function $H_{t, i}$ of a transitional layer under interface $i$ (Figure 1) is expressed as

$$
H_{t, i}(\omega)=\sum_{k=i}^{M-i}\left(\frac{\exp \left(-j \omega \tau_{k}\right)}{c_{1} \tau_{k}} V_{0, k} \prod_{m=2}^{k} H_{m}^{2} T_{0, m-1} T_{0, m-1}^{\prime}\right),
$$

where $V_{0, k}$ and $T_{0, k}$ represent the reflection and the transmission coefficients defined by the Snell-Descartes law for a smooth interface and $k$ and $m$ are the index of the layer and $m=M-i$. We define the thickness of a transitional layer in terms of oneway traveltime by $\Delta \tau=\tau_{m}-\tau_{i}$.

When an acoustic wave reaches a transitional layer between two media, the relative amplitude of the transmitted and reflected fields depends on the impedance contrast between the two media and the continuous impedance variation inside the transitional layer. For a thick transitional layer ( $\lambda \ll \Delta r$, where $\Delta r$ is the spatial thickness of the transitional layer), most of the acoustic field is transmitted into the structure. When the transitional layer is very thin $(\lambda \gg \Delta r)$, its impact tends to be transparent: 
Transmission and reflection depend only on the two media properties. For transitional layers with intermediate thickness $(\lambda / 4 \leq \Delta r \leq \lambda / 2)$, reflection is particularly affected (Ainslie, 2005). This case can occur with chirp sonars for transitional layer thicknesses varying from a few centimeters to a few decimeters. In this study, we are then concerned by transitional layers with thicknesses smaller than half a wavelength: $0 \leq \Delta r \leq \lambda / 2$, i.e., $0 \leq \Delta \tau \leq \pi / \omega$.

\section{Roughness}

The presence of rough interfaces causes scattering losses on the coherent propagation, which are accounted for through the coefficients of reflection and transmission. Under the assumption of a normally distributed random surface, roughness can be characterized by two parameters: the mean standard deviation of surface height $\sigma$ and the correlation length of the surface $\ell_{c}$. The hypothesis of a Gaussian distribution offers a good compromise between fitting the experiment and the complexity of the modeling. Given the monostatic configuration of chirp sonars close to normal incidence, no specific scattering direction is favored so that roughness with a specific orientation could also be approximated by a Gaussian distribution. Under the Kirchhoff assumption, $\ell_{c} / \lambda \gg 1$ (Thorsos, 1988), the spatial distribution has minor effects on the coherent acoustic field, which mainly depends on the standard deviation of roughness. In that regime, coherent reflection at normal incidence is given by (Eckart, 1953)

$$
\begin{gathered}
V_{i}=V_{0, i} e^{\frac{-Q_{R, i}^{2}}{2}}, \\
Q_{R, i}=2 k_{i} \sigma_{i},
\end{gathered}
$$

where $\sigma_{i}$ represents the roughness standard deviation, $k_{i}$ is the wave number, $c_{i}$ is the sound speed, and $Q_{R, i}$ is the Rayleigh parameter.
In contrast, as discussed in Appendix A, the coherent transmission is less sensitive than reflection to rough interfaces. The impact of roughness (Appendix A) can be neglected so that the transmission coefficient is suitably modeled by Snell-Descartes law, $T_{i}=T_{0, i}$.

Results from the scaled tank experiments, described in Appendix A, suggest that the effect of roughness on transmission can still be neglected even if the Kirchhoff assumption is not met $\left(\ell_{c} / \lambda \leq 1\right)$. However, the reflection coefficient deviates by almost $10 \mathrm{~dB}$ from the coherent reflection model, equation 8 , for fairly rough interfaces $\left(\ell_{c} / \lambda \leq 1\right)$.

\section{EXPERIMENTAL TESTING}

The forward modeling is now tested with in situ measurements from the Meriadzek Terrace in the northeast Atlantic using the equipment of the French research vessel Pourquoi Pas? from Institut Français de Recherche pour l'Exploitation de la Mer (IFREMER). This site is located in $2100 \mathrm{~m}$ of water in the northern part of the Bay of Biscay along the Celtic Margin (Bourillet et al., 2003). The region is characterized by low lateral variability with well-sorted fine-grained sediments (Zaragosi et al., 2001). In the operating frequency range of chirp sonars $(1-10 \mathrm{kHz})$, the interfaces are effectively smooth.

Core samples were recovered from this area during the Essai Carottage (ESCAROT) survey in March 2006 (Woerther et al., 2006) using the giant piston corer Calypso. Seismograms were collected during the Transit Valorisé Toulon-Brest (TVTB) survey in June 2007 with the chirp sonar Echoes 3500 emitting chirp pulses with a frequency modulation varying from 1.7 to $5.3 \mathrm{kHz}$. The KESC3-06 core examined in this paper is located $1 \mathrm{~km}$ away from the seismic profile. The core was recovered using settings that reduce the possible disturbances of the elastic recoil of the main cable and the piston (Bourillet et al., 2007).

Table 1. Attenuation of saturated sediment from the literature. Values in italics were computed by the authors. References are as follows: [1], Hamilton (1972); [2], McCann and McCann (1969); [3], Zimmer et al. (2010); [4], Lewis (1971); [5], Best et al. (2001);

\begin{tabular}{|c|c|c|c|c|c|}
\hline \multirow[b]{2}{*}{ Sediment type } & \multirow[b]{2}{*}{$k_{p}\left(\mathrm{~dB} / \mathrm{m} / \mathrm{kHz}^{n}\right)$} & \multirow[b]{2}{*}{$n$} & \multirow[b]{2}{*}{ Ref. } & \multicolumn{2}{|c|}{ Linear regression } \\
\hline & & & & $k_{p}^{\prime}(\mathrm{dB} / \mathrm{m} / \mathrm{kHz})$ & $b^{\prime}(\mathrm{dB} / \mathrm{m})$ \\
\hline Coarse sand & 0.93 & 0.96 & {$[1]$} & 0.48 & 0.09 \\
\hline Medium sand & $0.41 \pm 0.12$ & $1 \pm 0.14$ & {$[1]$} & $0.41 \pm 0.12$ & 0 \\
\hline \multirow[t]{2}{*}{ Fine sand } & 0.13 & $1.26 \pm 0.13$ & {$[2]$} & $0.25 \pm 0.08$ & -0.23 \\
\hline & $0.45 \pm 0.07$ & $1.04 \pm 0.07$ & {$[1]$} & $0.50 \pm 0.09$ & -0.08 \\
\hline \multirow[t]{3}{*}{ Very fine sand } & 0.27 & $1.17 \pm 0.13$ & {$[2]$} & $0.41 \pm 0.13$ & -0.27 \\
\hline & 0.56 & $1.00 \pm 0.01$ & {$[2]$} & 0.56 & 0 \\
\hline & $0.38 \pm 0.05$ & $1.11 \pm 0.06$ & [1] & $0.51 \pm 0.08$ & -0.22 \\
\hline Relatively homogeneous sand & $1.18-2.6$ & $0.57 \pm 0.05$ & [3] & 0.29 to 0.88 & $1.15-2.53$ \\
\hline Silt & 0.3 & $1.05 \pm 0.15$ & [2] & $0.34 \pm 0.13$ & -0.07 \\
\hline Clayey silt & 0.19 & 0.94 & {$[1]$} & 0.16 & 0.05 \\
\hline Sand-silt-clay & 0.393 & 0.99 & {$[4]$} & 0.38 & 0.02 \\
\hline Poorly sorted sediment & 1.46 & $0.63 \pm 0.33$ & {$[5]$} & $0.79 \pm 0.64$ & 1.34 \\
\hline Fine-grained unconsolidated sediment & 0.055 & 1.12 & {$[6]$} & 0.076 & -0.04 \\
\hline
\end{tabular}
[6], Bowles (1997). 
The recovered sediments present a maximal quality index (Woerther et al., 2006), meaning that the sediment suffered little deformation from the corer. Sound speed (Figure 2a) and density (Figure 2b) were measured for this sediment core with a multisensor core logger (Schultheiss and Weaver, 1992). The impedance profile for the first $15 \mathrm{~m}$ is plotted in Figure 2c.

The compressed synthetic seismogram, constructed from the impedance profile (Figure 2c) and the forward model, is presented Figure $3 b$. In the simulation, the effect of attenuation is also neglected along the first $15 \mathrm{~m}$ of the sediment sample. Chirp-sonar data from the TVTB survey show that the acoustic signal propagates into the subbottom sediment up to about 69 $\mathrm{ms}$, which corresponds to about $50 \mathrm{~m}$ with an approximate subbottom sound speed of $1480 \mathrm{~m} / \mathrm{s}$ (Rakotonarivo, 2009). From the active-sonar equation (Lurton, 2002), a rough estimate of the average attenuation for $50 \mathrm{~m}$ of propagation into the sediment is $k_{p} \approx 0.07 \mathrm{~dB} / \mathrm{m} / \mathrm{kHz}$ (Rakotonarivo, 2009).

The synthetic seismogram is compared to the coherent experimental seismogram in Figure $3 \mathrm{a}$ and $3 \mathrm{~b}$. The coherent experimental seismogram (Figure 3a) is obtained by spatially averaging seismograms over 30 successive pings. There is good agreement between the synthetic and experimental seismograms (Figure 3b). In particular, early arrivals from the time series (Figure 3b), which correspond to shallow layers, show good phase and amplitude agreements. However, there is a phase mismatch between synthetic and experimental data for later arrivals from the seismograms. Echoes from deeper layers occur about $1.4 \mathrm{~ms}$ earlier in the synthetic seismogram than in the experimental seismogram (Figure 3b). One explanation for this shift is core deformations caused by behavior of the piston during coring. The gravity piston corer works as a syringe so that it encounters either an oversampling or an undersampling of sediment (Skinner and McCave, 2003). Those deformations can cause a shift of more than 5-6 ms on the seismogram peaks (Széréméta et al., 2004). Assuming that core deformations are the main reason for these small peak shifts, the effect of core deformations can be treated as a monotonic dilatation function on the core distances. On the basis of the shift between echoes from the synthetic and the experimental seismograms (Figure $3 b$ ), we modify the depth dependence of measured sound speed and density. Then, we compute another compressed seismogram from the sound speed and density profiles using the modified distances (Figure 3c). As a result, the main echoes of the experimental and the new synthetic seismograms match better for amplitude and phase (Figure 3c). The matching of the main echoes locations (Figure $3 \mathrm{c}$ ) provides the deformation parameters.

Despite the inherent experimental uncertainties, the degree of agreement between the experimental data and the synthetic data is encouraging. It suggests that inversion of chirp-sonar data using the presented forward modeling is potentially possible for many sediment layers.

\section{SENSITIVITY ANALYSIS}

In the present study, five sediment parameters can potentially be estimated from chirp-sonar data: the layer location in terms of two-way traveltime, the impedance, the attenuation coefficient, the roughness standard deviation, and the thickness of a transitional layer. The estimation of the sediment parameters is achieved by minimizing the cost function,

$$
E\left(\tau, \mathbf{Z}, \mathbf{k}_{\mathbf{p}}, \sigma, \Delta \tau\right)=\frac{\int_{t_{0}}^{t_{f}} d t\left|\gamma\left(\tau, \mathbf{Z}, \mathbf{k}_{\mathbf{p}}, \sigma, \Delta \tau\right)-\gamma_{\mathrm{obs}}\right|}{\int_{t_{0}}^{t_{f}} d t\left|\gamma_{\mathrm{obs}}\right|},
$$

where the lower and upper time limits, $t_{0}$ and $t_{f}$, delimit the portion of the seismogram to be inverted, $\gamma_{\text {obs }}$ refers to the seismogram measured by the chirp sonar, $\gamma$ is the synthetic seismogram calculated from the forward modeling, and sediment parameters $\tau, \mathbf{Z}, \mathbf{k}_{\mathbf{p}}, \boldsymbol{\sigma}, \boldsymbol{\Delta} \tau$ are vectors of size $n$, where $n$ is the number of detected layers. The cost function, equation 9, uses the norm $L_{1}$ to favor robustness of the inversion (Tarantola, 2005).
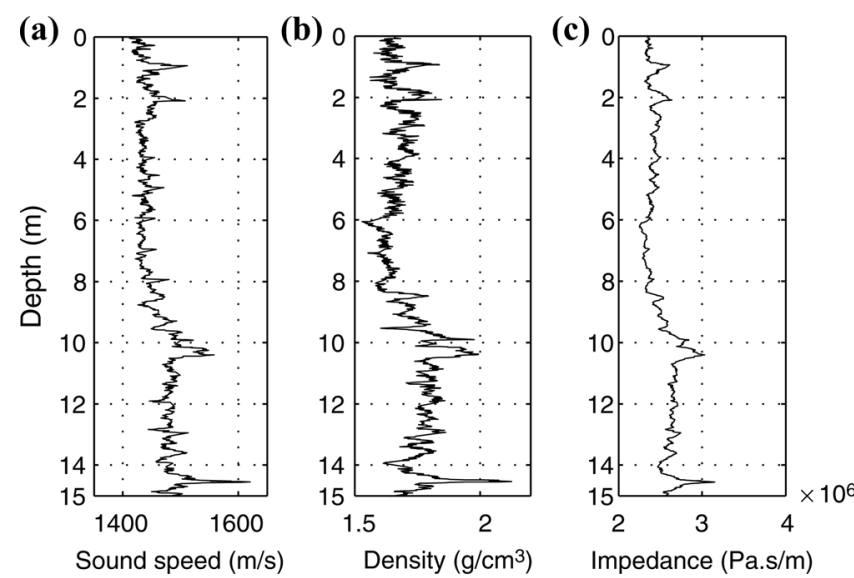

Figure 2. Properties measured along the KESC3-06 core sample at $4^{\circ}$ C. (a) Sound speed profile with an uncertainty of $10 \mathrm{~m} / \mathrm{s}$. (b) Density profile with an uncertainty of $0.5 \mathrm{~g} / \mathrm{cm}^{3}$. (c) Impedance profile.
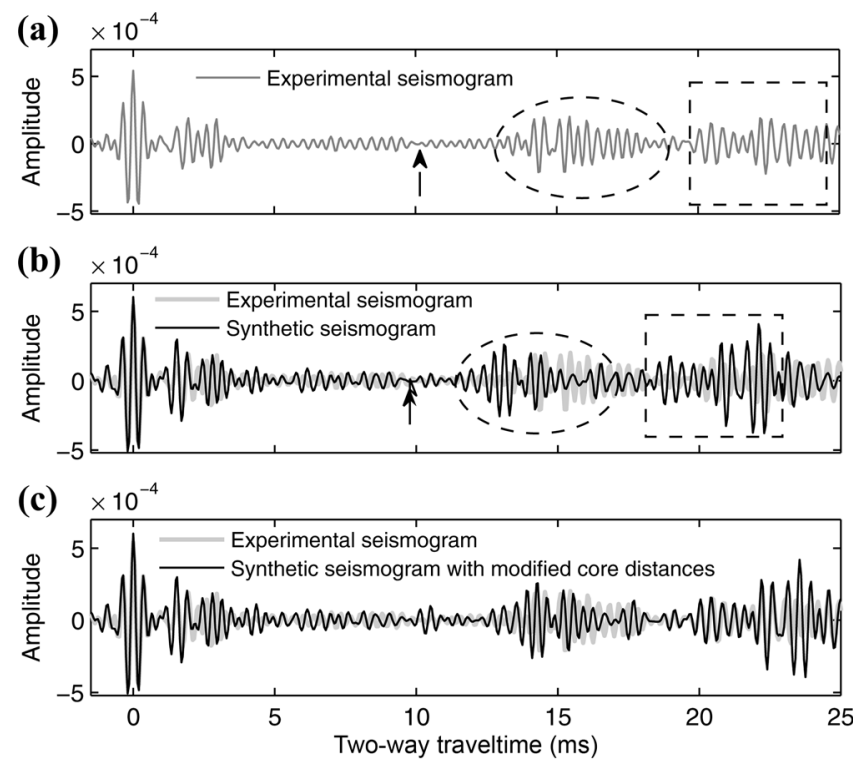

Figure 3. (a) Seismogram from chirp-sonar measurements. (b) Comparison of the experimental and synthetic seismograms without core distance compensation, The arrows, dashed ellipses, and dashed rectangles show the similarities between the two seismograms; the time shift between the two dashed ellipses is about $1.4 \mathrm{~ms}$. (c) Comparison of the experimental and synthetic seismograms with modified core distances. 
To determine the extent to which sediment parameters can be estimated from data inversion, we conduct a sensitivity analysis for the two subbottom sediment scenarios described in Figure 4. The study is performed at two frequency regimes on each layer of the tested sediment configurations. The first configuration consists of an attenuating, layered medium with rough interfaces and a transitional layer. The objective is to study the impact of roughness and the transitional layer separately and the influence of attenuation. The second scenario examines the simultaneous influence of roughness and continuous impedance variations. The sensitivity study is investigated for frequencies varying from 1.7 to $5.2 \mathrm{kHz}$ and for the frequency bandwidth ranging from 5 to $10 \mathrm{kHz}$. The transitional layer thickness is about $\Delta r \approx \lambda / 8$ (i.e., $\Delta \tau=35 \mu \mathrm{s}$ ) at the central frequency $f_{c}=3.5$ $\mathrm{kHz}$ and $\Delta r \approx \lambda / 4$ (i.e., $\Delta \tau=33 \mu$ s) at $f_{c}=7.5 \mathrm{kHz}$.

For each subbottom sediment scenario and each parameter, we calculate the output of the cost function, equation 9, on each layer. We vary each parameter independently for all values of the parameter space given in Table 2, while all other sediment parameters remain fixed. The cost function equals zero when the steering value fits the real value of the studied parameter. This

\begin{tabular}{|c|c|c|c|c|}
\hline & 1 & Parameters & 2 & Parameters \\
\hline $\begin{array}{ll}\text { Layer } 1 & \\
\text { water } & \Xi \\
& \stackrel{\sim}{N}\end{array}$ & & $\begin{array}{l}\rho=1 \mathrm{~g} / \mathrm{cm}^{3} \\
c=1500 \mathrm{~m} / \mathrm{s} \\
\sigma=2.3 \mathrm{~cm}\end{array}$ & & $\begin{array}{l}\rho=1 \mathrm{~g} / \mathrm{cm}^{3} \\
c=1500 \mathrm{~m} / \mathrm{s} \\
\sigma=2.3 \mathrm{~cm}\end{array}$ \\
\hline $\begin{array}{ll}\text { Layer } 2 & \\
& \varepsilon \\
& \mathcal{N}\end{array}$ & & $\begin{array}{l}\rho=1.3 \mathrm{~g} / \mathrm{cm}^{3} \\
c=1600 \mathrm{~m} / \mathrm{s}\end{array}$ & $\begin{array}{l}E \\
E \\
\text { ñ } \\
\circ \downarrow\end{array}$ & $\begin{array}{l}\rho=1 \text { to } 1.3 \mathrm{~g} / \mathrm{cm}^{3} \\
c=1600 \mathrm{~m} / \mathrm{s} \\
\rho=1.3 \mathrm{~g} / \mathrm{cm}^{3} \\
c=1600 \mathrm{~m} / \mathrm{s}\end{array}$ \\
\hline Layer 3 个 & $\begin{array}{ll}E \uparrow \\
8 \\
0\end{array}$ & $\begin{array}{l}\rho=1.3 \text { to } 1.8 \mathrm{~g} / \mathrm{cm}^{3} \\
c=1725 \mathrm{~m} / \mathrm{s} \\
\rho=1.8 \mathrm{~g} / \mathrm{cm}^{3} \\
c=1725 \mathrm{~m} / \mathrm{s} \\
K_{\mathrm{p}}=0.1 \mathrm{~dB} / \mathrm{m} / \mathrm{KHZ} \\
\sigma=2 \mathrm{~cm}\end{array}$ & & $\begin{array}{l}\rho=1.8 \mathrm{~g} / \mathrm{cm}^{3} \\
c=1725 \mathrm{~m} / \mathrm{s} \\
K_{\mathrm{p}}=0.1 \mathrm{~dB} / \mathrm{m} / \mathrm{KHZ} \\
\sigma=2 \mathrm{~cm}\end{array}$ \\
\hline Layer 4 & & $\begin{array}{l}\rho=2 \mathrm{~g} / \mathrm{cm}^{3} \\
c=2200 \mathrm{~m} / \mathrm{s}\end{array}$ & & $\begin{array}{l}\rho=2 \mathrm{~g} / \mathrm{cm}^{3} \\
c=2200 \mathrm{~m} / \mathrm{s}\end{array}$ \\
\hline
\end{tabular}

Figure 4. Schematic summary of the physical properties of the subbottom sediments tested in the sensitivity analysis.

Table 2. Searching intervals of sediment parameters defined from the forward modeling analysis. The layer location searching area is given by the two-way traveltime of the echo peak at time $\tau$ and the match-filtered signal resolution, which is approximately $1 / B_{w}$, where $B_{w}$ is the bandwidth of the transmitted chirp.

\begin{tabular}{lcc} 
Parameters & Lower bound & Upper bound \\
\hline Two-way traveltime & $\tau-2 / B_{w}$ & $\tau+2 / B_{w}$ \\
$Z(\mathrm{~Pa}-\mathrm{s} / \mathrm{m})$ & $150 \times 10^{4}$ & $378 \times 10^{4}$ \\
$k_{p}(\mathrm{~dB} / \mathrm{m} / \mathrm{kHz})$ & 0 & 0.8 \\
$\sigma(\mathrm{cm})$ & 0 & 2.5 \\
$\Delta \tau(\mathrm{s})$ & 0 & $\pi / \omega$ \\
\hline
\end{tabular}

analysis is conducted for all five sediment parameters in each layer at the two frequency regimes. Figures 5 and 6 plot the results for the low-frequency chirp $\left(B_{w}=1.7-5.2 \mathrm{kHz}\right)$ and the high-frequency chirp $\left(B_{w}=5-10 \mathrm{kHz}\right)$ on both subbottom scenarios, respectively.
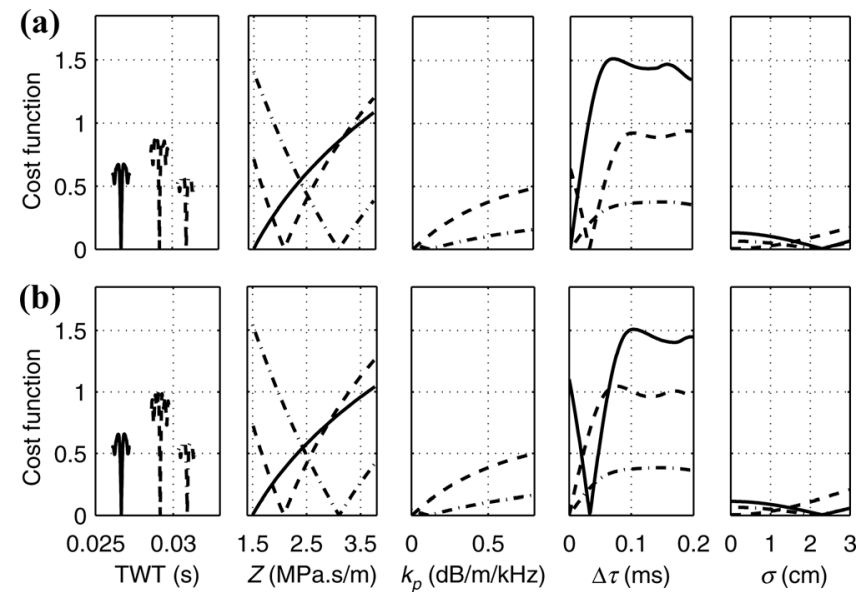

$\begin{array}{lllllll}0 & 0.1 & 0.2 & 0 & 1 & 0 & 3\end{array}$

$\Delta \tau(\mathrm{ms})$

Figure 5. Sensitivity analysis for the chirp with $B^{w}=1.7-5.2 \mathrm{kHz}$ for the two subbottom layouts given in Figure 4: (a) scenario 1 and (b) scenario 2 . The cost function is calculated for each sediment parameter in the first layer (solid lines), the second layer (dashed lines), and the third layer (dash-dotted lines).
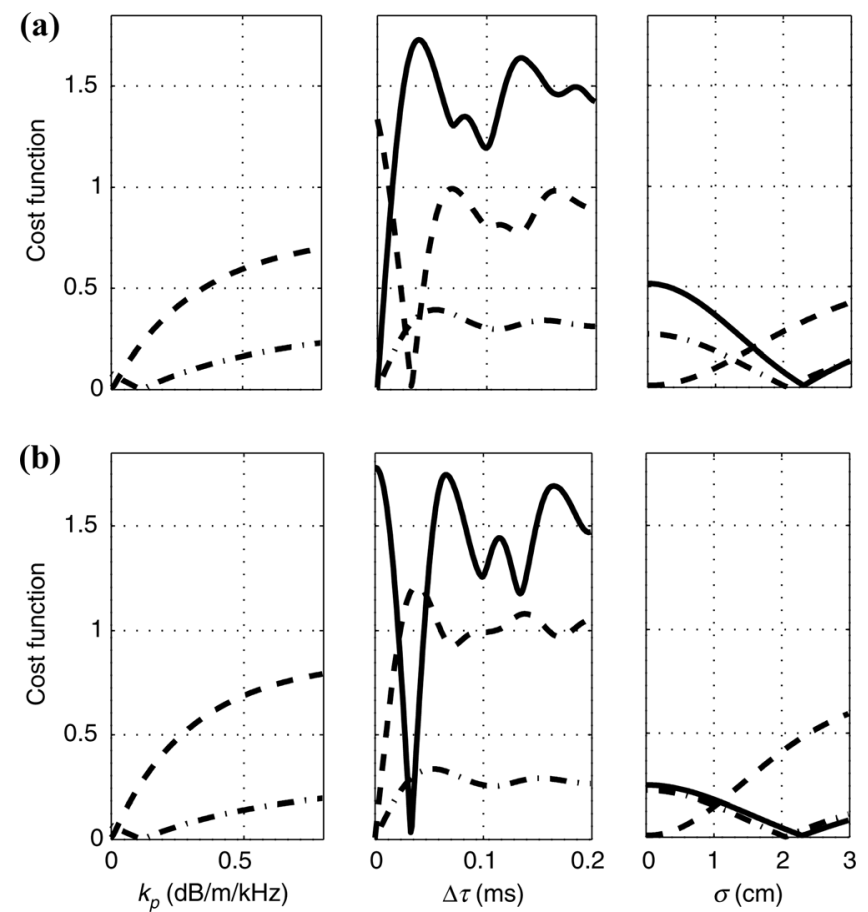

Figure 6. Sensitivity analysis for the chirp with $B^{w}=5-10 \mathrm{kHz}$ for the two subbottom layouts given in Figure 4: (a) scenario 1 and (b) scenario 2. The cost function is calculated for each sediment parameter in the first layer (solid lines), the second layer (dashed lines), and the third layer (dash-dotted lines). 
Analysis of Figure 5 shows that cost functions related to the layer position, the impedance, and the transitional layer thickness have higher dynamic range than cost functions from attenuation and roughness standard deviation. Consequently, estimations of impedance profile and transitional layer thickness appear more robust than estimating roughness and attenuation for this study case. When the depth layer increases or the frequency decreases, the estimations of attenuation, transitional layer, and roughness become less efficient and less robust. Indeed, the dynamic range of their cost functions decreases at lower frequencies (Figure 6) or/and for deeper sediment layers into the subbottom sediment (Figures 5 and 6).

Given the low value of the cost function in Figure 5 for the low-frequency chirp, the impact of roughness can be neglected against the other sediment parameters for frequencies below 5 $\mathrm{kHz}$. At higher frequencies (Figure 6), its cost function has a higher dynamic range, so that an estimate of the roughness standard deviation seems possible for the first interface. We also note that the influence of roughness becomes stronger when the impedance contrast at the rough interface is stronger. When the rough interface overlaps a transitional layer, the cost function of the former is affected, whereas the cost function of the latter remains almost constant (Figure $5 \mathrm{a}$ and $5 \mathrm{~b}$ ). Thus, at a layer with simultaneous effects of rough interface and transitional layer, the latter prevails over the impact of roughness. Consequently, either roughness or transitional layer can prevail on the reflected echo.

\section{DISCUSSION}

The sensitivity analysis suggests that the inversion method may be more robust for estimating layer locations, impedance, and transitional layer thickness. At frequencies less than $5 \mathrm{kHz}$, the impact of roughness is negligible as compared to the other sediment parameters. So this reduces by one the number of parameters that must be estimated. At frequencies higher than 5 $\mathrm{kHz}$, the extraction of roughness is more consistent for the upper interface than for underlying layers. Also, its influence cannot be distinguished from a transitional layer, so that either a rough interface or a continuous impedance can prevail on the reflected echo. Thus, the inversion process consists of estimating layer locations, impedance, attenuation, and either roughness or transitional layer thickness.

Estimation of attenuation depends on the values of the coefficient $k_{p}$ and the thickness of the layer that we integrate to estimate attenuation. In the presented examples, the impact of attenuation is not very strong due to the small thickness of the layer. Given the maximal value of attenuation in this study, $k_{p}=0.8 \mathrm{~dB} / \mathrm{kHz} / \mathrm{m}$, the latter causes a $3-\mathrm{dB}$ loss for layer thicknesses greater than $2 \lambda$.

Estimating sediment parameters simultaneously on many layers would overcome the difficulties that arise from the simultaneous influences of attenuation with either roughness or transitional layers. Indeed, the attenuation of a given layer governs the waveform of echoes from all underlying layers, whereas roughness and transitional layer thickness impact only locally on the reflection. Thus, the distinct influence of each parameter can potentially be achieved.

Also, the discussion of roughness emphasizes that the reflection coefficient can deviate by almost $10 \mathrm{~dB}$ from the coherent reflec- tion model, equation 8 , when the Kirchhoff assumption is not met. At the frequencies of chirp sonars, the wavelength in seawater varies from $0.15 \mathrm{~m}$ at $10 \mathrm{kHz}$ to $1.5 \mathrm{~m}$ at $1 \mathrm{kHz}$. In a shallow-water environment, Briggs (1989) measured roughness varying from 0 to $2.3 \mathrm{~cm}$. For frequencies close to $10 \mathrm{kHz}$ and for sandy bottoms with $\sigma \approx 2 \mathrm{~cm}$ (Briggs, 1989), the possibility of having a wavelength in the same order as the correlation length and a Rayleigh parameter close to unity can occur. Nevertheless, it is not possible to determine a priori the validity or nonvalidity of the Kirchhoff assumption from chirp-sonar data themselves. If these hypotheses are not met, the forward model will provide a biased estimate of the echo reflected by a rough interface. However, echoes from underlying interfaces will be suitably modeled as transmission is not affected much by roughness.

\section{CONCLUSIONS}

This paper presented the forward modeling of chirp-sonar data as a basis for subbottom sediment characterization. The method is based on a transfer function approach and includes the effects of layer thickness, impedance mismatch, attenuation, and the impact of roughness and transitional layers. In spite of experimental uncertainties, model testing with chirp-sonar data from a deep-sea environment shows good agreement for the first $15 \mathrm{~m}$ of buried sediments. Then, a frame for the future inversion using this forward modeling was examined. We have specified the cost function, based on the $L_{1}$ norm, and the parameter space that will be used for the inversion. Sensitivity analysis suggests that the estimation of layer thickness, impedance, and transitional layer thickness is more robust than the conditional estimations of roughness and attenuation. Roughness can be estimated for frequencies above $5 \mathrm{kHz}$, and effective attenuation can be extracted from chirp-sonar data for layers thicker than $2 \lambda$. Also, estimations of frequency-dependent parameters (attenuation, transitional layers, and roughness) become less efficient for deeper sediment layers under the seawater-sediment interface. A particular inversion would consist of estimating sediment parameters linked to either (1) impedance, attenuation, and roughness or (2) impedance, attenuation, and transitional layers. The inversion of chirpsonar data will be examined in a future paper.

\section{ACKNOWLEDGMENTS}

This work was funded by Ixsea and the Association Nationale de la Recherche et de la Technologie (ANRT). The authors would like to thank the reviewers for their helpful comments.

\section{APPENDIX A}

\section{ROUGHNESS EXPERIMENTS}

The goal of this appendix is to experimentally test the model of coherent reflection and transmission given in equations 8 and A-1 and to examine the impact of roughness on the coherent wave propagation when the Kirchhoff assumption is and is not valid. Under the Kirchhoff assumption, the coherent transmission at normal incidence is given by (Nagy and Adler, 1987)

$$
T_{i}=T_{0, i} e^{\frac{-Q_{T, i}^{2}}{2}}
$$




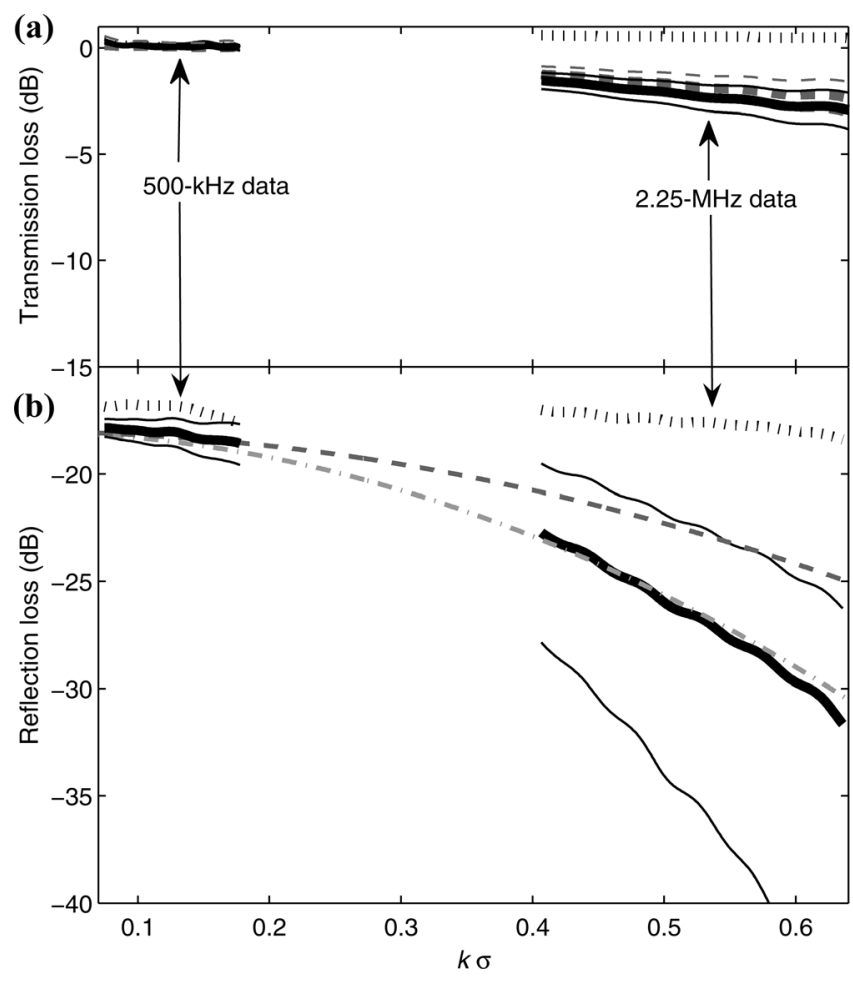

Figure A-1. (a) Transmission loss measured through the rough silicon plate in the forward (thick solid lines) and the backward (thick dashed lines) directions and through the smooth silicon plate (thick dotted lines). (b) Reflection loss measured on the rough face (thick solid lines) and the smooth surface (thick dotted lines) of the silicon plate. The measured reflection losses are compared to the model of coherent reflection coefficient given in equation 8 (thick dashed lines), and the thick dash-dotted line represents the best-fit curves with measured data. In (a) and (b), the thin lines represent the standard deviation of measured reflection data.

$$
Q_{T, i}=k_{i+1} \sigma_{i}\left(\frac{c_{i+1}}{c_{i}}-1\right),
$$

where $Q_{T, i}$ is the Roughness parameter in transmission. Scaled tank experiments are conducted on a rough calibrated plate to study the case where $\ell_{c} / \lambda \leq 1$ with small $\left(Q_{R} \ll 1\right)$ and fair $\left(Q_{R} \geq 1\right)$ roughness. Reflection and transmission measurements are made on a calibrated silicon plate characterized by an impedance $Z=11.6 \times 10^{5} \mathrm{~Pa}-\mathrm{m} / \mathrm{s}$ to reproduce the low-impedance constrasts in soft marine sediment. One side of the plate is smooth, and the other side has a rough surface with a Gaussian spectrum, a correlation length $\ell_{c} \approx 0.77 \mathrm{~mm}$, and a roughness standard deviation $\sigma=0.06 \mathrm{~mm}$. Measurements are made at 500 $\mathrm{kHz}(\lambda \approx 3 \mathrm{~mm})$ to reproduce $\ell_{c} / \lambda \geq 1$ and small roughness $\left(Q_{R} \approx 0.25\right)$ and at $2.25 \mathrm{MHz}(\lambda \approx 0.67 \mathrm{~mm})$ to reproduce $\ell_{c} / \lambda \geq 1$ and fair roughness $\left(Q_{R} \approx 1.13\right)$. Reflected fields and backward and forward transmitted fields are recorded for 50 positions on the rough plate. Coherent reflections and double transmissions are derived from the coherent summation of the acoustic field over the 50 realizations. Following the same experimental method, those parameters are also derived from measurements on a reference silicon plate with a smooth surface on each side and with the same impedance features as the rough plate. The term double transmission refers to transmission through both sides of the tested plate. Transmission loss on the latter is plotted in Figure A-1a. Coherent transmissions, measured in the forward and backward directions, deviate from each other by less than $0.5 \mathrm{~dB}$ for $Q_{R} \ll 1$ and $Q_{R} \leq 1$ (Figure A-1a). Given the accuracy of the experiment (at least $1 \mathrm{~dB}$ ), we can consider that the impact of roughness on transmission is identical for the forward and backward directions. Coherent reflection (Figure A-1b) and transmission (Figure A-1a) losses increase with frequency, as scattering is more significant at higher frequencies. Coherent reflected fields are affected by $10-\mathrm{dB}$ scattering loss, which is much more than the 2-dB loss for coherent transmitted fields. The impact of scattering on acoustic transmission can be neglected even if the ratio $\ell_{c} / \lambda$ and Rayleigh parameter are close to or greater than 1 . As seen in Figure A-1a, there is less than a 3-dB loss. Therefore, the transmission coefficient from equation A-1 is well approximated by the Snell-Descartes law.

Furthermore, reflection loss (Figure A-1b) is well predicted by equation 8 for measurements at $500 \mathrm{kHz}$, even if $\ell_{c} / \lambda \leq 1$. As scattering loss is very low in comparison to the accuracy of the experiment, the reflection coefficient is defined by the Snell-Descartes law for $Q_{R} \ll 1$. At $2.25 \mathrm{MHz}\left(Q_{R} \approx 1.13\right)$, reflection loss (Figure A-1b) deviates by almost $10 \mathrm{~dB}$ from the coherent reflection model given in equation 8 . Scattering losses are greater here due to the relative size of the wavelength compared to the correlation length, which may favor interferences between specular and scattered components in the return signal. If this happens, the coherent reflection coefficient defined in equation 8 is not valid. Noting that reflection loss follows a decreasing trend similar to the one predicted by theory (equation 8 ), the latter model is extended to fit the experimental curves when $\ell_{c} / \lambda \leq 1$ (Figure A1b). The expression of the Rayleigh parameter from expression 8 is modified, and a better fit is obtained for

$$
Q_{R}^{\prime}=2.67 k \sigma
$$

where $Q_{R}^{\prime}$ is the modified empirical parameter at normal incidence.

\section{REFERENCES}

Ainslie, M. A., 2005, The effect of centimeter scale impedance layering on the normal-incident seabed reflection coefficient, in Boundary influences in high frequency shallow water acoustics: University of Bath Press, 423-430.

Best, E. I., Q. J. Huggett, and A. J. K. Harris, 2001, Comparison of in situ and laboratory acoustic measurements on Lough Hyne marine sediments: The Journal of the Acoustical Society of America, 110, 695709.

Bourillet, J.-F., G. Damy, S. Migeon, N. Sultan, and L. Dussud, 2007, Behaviour of a piston corer from accelerometers and new insights on associated disturbances: Presented at the 6th international conference on Offshore Site Investigation and Geotechnics, Society for Underwater Technology.

Bourillet, J.-F., J.-Y. Reynaud, A. Baltzer, and S. Zaragosi, 2003, The 'fleuve manche': The submarine sedimentary features from the outer shelf to the deep-sea fans: Journal of Quaternary Science, 18, 261-282.

Bowles, F. A., 1997, Observations on attenuation and shear-wave velocity in fine-grained, marine sediments: The Journal of the Acoustical Society of America, 101, 3385-3397, doi:10.1121/1.419374.

Brekhovskikh, L. M., and Y. P. Lysanov, 1991, Fundamentals of ocean acoustics, 2nd ed.: Springer-Verlag.

Briggs, K. B., 1989, Microtopographical roughness of shallow-water continental shelves: IEEE Journal of Oceanic Engineering, 14, 360-367, doi:10.1109/48.35986.

Buckingham, M. J., 2007, On pore fluid viscosity and the wave properties of saturated granular materials including marine sediments: The Journal of the Acoustical Society of America, 122, 1486-1501, doi:10.1121/ 1.2759167 . 
Bull, J. M., R. Quinn, and J. Dix, 1998, Reflection coefficient calculation from marine high resolution seismic reflection data and application to an archaeological case study: Marine Geophysical Researches, 20 1-11, doi:10.1023/A:1004373106696.

Chotiros, N. P., and M. J. Isakson, 2004, A broadband model of sandy ocean sediments: Biot-Stoll with contact squirt flow and shear drag: The Journal of the Acoustical Society of America, 116, 2011-2022, doi:10.1121/1.1791715.

DeBruin, D. L., 1995, Classification of marine sediment using fuzzy logic impedance model inversion: Ph.D. thesis, Florida Atlantic University.

Eckart, C., 1953, The scattering of sound from the sea surface: The Journal of the Acoustical Society of America, 25, 566-570, doi:10.1121/ 1.1907123 .

Gutowski, M., J. Bull, T. Henstock, J. Dix, P. Hogarth, T. Leighton, and P. White, 2002, Chirp sub-bottom profiler source signature design and field testing: Marine Geophysical Researches, 23, 481-492, doi:10.1023/B:MARI.0000018247.57117.0e.

Hamilton, E. L., 1970, Reflection coefficients and bottom losses at normal incidence computed from Pacific sediment properties: Geophysics, 35, 995-1004, doi:10.1190/1.1440149.

1972, Compressional-wave attenuation in marine sediments: Geophysics, 37, 620-646, doi:10.1190/1.1440287.

Hamilton, E. L., and R. T. Bachman, 1982, Sound velocity and related properties of marine sediments: The Journal of the Acoustical Society of America, 72, 1891-1904, doi:10.1121/1.388539.

Hardage, B. A., P. Murray, D. Sava, M. M. Backus, R. Remington, R. Graebner, and H. H. Roberts, 2006, Evaluation of deepwater gas-hydrate systems: The Leading Edge, 25, 572-576, doi:10.1190/1.2202661.

Ivakin, A. N., and D. R. Jackson, 1998, Effects of shear elasticity on sea bed scatterings: Numerical examples: The Journal of the Acoustical Society of America, 103, 346-354, doi:10.1121/1.421094.

Jacobson, R. S., 1987, An investigation into the fundamental relationships between attenuation, phase dispersion, and frequency using seismic refraction profiles over sedimentary structures: Geophysics, 52, 72-87, doi:10.1190/1.1442242.

Kibblewhite, A. C., 1989, Attenuation of sounds in marine sediments: A review with emphasis on a new low-frequency data: The Journal of the Acoustical Society of America, 86, 716-737, doi:10.1121/1.398195.

Langli, B., and J. C. LeGac, 2004, The first results with a new multibeam subbottom profiler, in Ocean '04 MTS/IEEE Techno-Ocean '04: IEEE, 1147-1153.

LeBlanc, L. R., L. Mayer, S. Panda, S. G. Schock, and J. King, 1992a, Sonar attenuation modeling for classification of marine sediments: The Journal of the Acoustical Society of America, 91, 116-126, doi: $10.1121 / 1.402759$.

LeBlanc, L. R., L. Mayer, M. Rufino, S. G. Schock, and J. King, 1992b, Marine sediment classification using chirp sonar: The Journal of the Acoustical Society of America, 91, 107-115, doi:10.1121/1.402758.

Lewis, L. F., 1971, An investigation of ocean sediments using the deep ocean sediment probe: Ph.D. thesis, University of Rhode Island.

Lurton, X., 2002, Underwater acoustic mapping systems, in An introduction to underwater acoustics: principles and applications: Springer, 255-256.

McCann, C., and D. M. McCann, 1969, The attenuation of compressional waves in marine sediments: Geophysics, 34, 882-892, doi:10.1190/ 1.1440059.

Nagy, P. B., and L. Adler, 1987, Surface roughness induced attenuation of reflected and transmitted ultrasonic waves: The Journal of the Acoustical Society of America, 82, 193-197, doi:10.1121/1.395545.

Panda, S., L. R. LeBlanc, and S. G. Schock, 1994, Sediment classification based on impedance and attenuation estimation: The Journal of the Acoustical Society of America, 96, 3022-3035, doi:10.1121/1.411266.
Pinson, L. J. W., T. J. Henstock, J. K. Dix, and J. M. Bull, 2008, Estimating quality factor and mean grain size of sediments from high-resolution marine seismic data: Geophysics, 73, no. 4, G19-G28, doi:10.1190/ 1.2937171

Rakotonarivo, S. T., 2009, Caractérisation quantitative des fonds marins à l'aide des sondeurs de sédiment $(1 \mathrm{kHz}-10 \mathrm{kHz})$ : Modélisation directe pour l'inversion sur données réelles: Ph.D. thesis, Université de Bretagne Occidentale.

Schock, S. G., 2004a, A method for estimating the physical and acoustic properties of the sea using chirp sonar data: IEEE Journal of Oceanic Engineering, 29, 1200-1217, doi:10.1109/JOE.2004.841421.

Schock, S. G., 2004b, Remote estimates of physical and acoustic sediment properties in the South China Sea using chirp sonar data and the Biot model: IEEE Journal of Oceanic Engineering, 29, 1218-1230, doi:10.1109/JOE.2004.842253.

Schock, S. G., L. R. LeBlanc, and L. A. Mayer, 1989, Chirp subbottom profiler for quantitative sediment analysis: Geophysics, 54, 445-450, doi:10.1190/1.1442670.

Schultheiss, P. J., and P. P. E. Weaver, 1992, Multi-sensor core logging for science and industry, in Oceans 92: Mastering the Oceans Through Technology: IEEE, vol. 2, 608-613.

Skinner, L. C., and I. N. McCave, 2003, Analysis and modelling of gravity and piston coring based on soil mechanics: Marine Geology, 199, 181204, doi:10.1016/S0025-3227(03)00127-0.

Stevenson, I. R., C. McCann, and P. B. Runciman, 2002, An attenuationbased sediment classification technique using Chirp sub-bottom profiler data and laboratory acoustic analysis: Marine Geophysical Research, 23, 277-298, doi:10.1023/A:1025708024518.

Stoll, R. D., 1977, Acoustic waves in ocean sediments: Geophysics, 42, 715-725, doi:10.1190/1.1440741.

Stoll, R. D., and G. M. Bryan, 1979, Physical properties of sediments containing gas hydrates: Journal of Geophysical Research, 84, 629-1634, doi:10.1029/JB084iB04p01629.

Széréméta, N., F. Bassinot, Y. Balut, L. Labeyrie, and M. Pagel, 2004 Oversampling of sedimentary series collected by giant piston corer: Evidence and corrections based on 3.5-khz chirp profiles: Paleoceanography, 19, PA1005, doi:10.1029/2002PA000795.

Tarantola, A., 2005, Least-absolute-values criterion and minimax criterion, in Inverse problem theory and methods for model parameter estimation: SIAM, 89-95.

Theuillon, G., Y. Stephan, and A. Pacault, 2008, High-resolution geoacoustic characterization of the seafloor using a subbottom profiler in the Gulf of Lion: IEEE Journal of Oceanic Engineering, 33, 240-254, doi:10.1109/JOE.2008.926958.

Thorsos, E. I., 1988, The validity of the Kirchhoff approximation for rough surface scattering using a Gaussian roughness spectrum: The Journal of the Acoustical Society of America, 83, 78-92, doi:10.1121/ 1.396188 .

Woerther, P. E., E. L. Breton, and R. Apprioual, 2006, Compte rendu de la mise en oeuvre des capteurs de pression et des accéléromètres sur le carottier Calypso du n/o Pourquoi Pas?: Mission ESCAROT 3: Technical report, IFREMER.

Zaragosi, S., F. Eynaud, C. Pujol, G. A. Auffret, J.-L. Turon, and T. Garlan, 2001, Initiation of the European deglaciation as recorded in the northwestern Bay of Biscay slope environments (Meriadzek terrace and Trevelyan escarpment): A multi-proxy approach: Earth and Planetary Science Letters, 188, 493-507, doi:10.1016/S0012-821X(01) 00332-6.

Zimmer, M. A., L. D. Bibee, and M. D. Richardson, 2010, Measurement of the frequency dependence of the sound speed and attenuation of seafloor sands from 1 to $400 \mathrm{kHz}$ : IEEE Journal of Oceanic Engineering, 35, 538-557, doi:10.1109/JOE.2010.2056230. 